Бальчугов Алексей Валерьевич, д.Т.Н., профессор кафедры МАХП, Ангарский государственный технический университет, РАНДОМИЗАЦИЯ ЭКСПЕРИМЕНТОВ В ХИМИЧЕСКОЙ ТЕХНОЛОГИИ e-mail: balchug@mail.ru

\title{
RANDOMIZATION OF EXPERIMENTS IN CHEMICAL TECHNOLOGY
}

\begin{abstract}
Аннотация. Показано, что рандомизация позволяет получить более достоверные результаты при экспериментальном исследовании гидродинамических, теплообменных и массообменных процессов.

Ключевые слова: рандомизация, экспериментальные исследования, систематическая погрешность.

Abstract. It is shown that randomization allows obtaining more reliable results in the experimental study of hydrodynamic, heat transfer and mass transfer processes.

Keywords: randomization, experimental studies, systematic error.

Рандомизация - это прием, который позволяет в некоторой степени компенсировать систематические погрешности при проведении эксперимента [1]. Он состоит в том, что запланированные опыты проводят в случайной последовательности, которая устанавливается либо с помощью таблицы случайных чисел, либо жеребьевкой. Например, необходимо рандомизировать во времени шесть опытов, обозначенных цифрами I, II,.., VI. Из таблицы случайных чисел возьмем из любой строки или любого столбца 6 последовательных чисел и соотнесем их с номерами опытов. Если при этом встречаются повторяющиеся числа, их отбрасывают. Так, опытам можно присвоить следующие новые случайные номера, указанные в таблице.
\end{abstract}

Таблица

Результаты рандомизации

\begin{tabular}{|c|c|c|}
\hline $\mathrm{I}-16$ & $\mathrm{III}-79$ & $\mathrm{~V}-61$ \\
\hline $\mathrm{II}-07$ & $\mathrm{IV}-57$ & $\mathrm{VI}-42$ \\
\hline
\end{tabular}

В соответствии с новыми номерами последовательность проведения опытов будет следующей: II, I, VI, IV, V, III.

Прием рандомизации имеет большое значение при планировании экспериментов в химической технологии. Например, при определении зависимости гидравлического сопротивления слоя орошаемой насадки от скорости газа, необходимо определить гидравлическое сопротивление слоя орошаемой насадки при постоянном расходе орошения и при следующих скоростях газа: 0,1 м/с; 0,5 м/с; 1,0 м/с; 1,5 м/с; 2 м/с; 2,5 м/с. Если опыты проводить последовательно в порядке возрастания скорости газа, то это может повлиять на характер перераспределения жидкости и газа в слое насадки, и, следовательно, внести сис- 
тематическую погрешность в результаты измерения. В соответствии с табл. 1 порядок проведения экспериментов должен быть следующий: 0,5 м/c; 0,1 м/с; 2,5 м/с; 1,5 м/с; 2 м/с; 1,0 м/с.

Аналогично прием рандомизации целесообразно использовать при проведении экспериментов по определению коэффициента теплопередачи в одноходовом кожухотрубчатом теплообменнике. Если в ходе экспериментов определяется зависимость коэффициента теплопередачи от скорости одного из теплоносителей, то последовательность проведения экспериментов необходимо определять также с помощью таблицы случайных чисел. Проводить эксперименты при постепенном увеличении скорости теплоносителя неправильно, поскольку это может повлиять на перераспределение жидкости по трубкам теплообменника и внести систематическую погрешность в результаты измерений.

Рандомизация оказывается плодотворной также при исследовании распределения концентрации компонента в жидкости по высоте тарельчатой массообменной колонны в установившемся режиме. Если отбирать пробы жидкости для химического анализа с каждой тарелки последовательно, начиная с верхней тарелки, то, в случае колебаний расхода жидкости или непроизвольного постепенного изменения начальной концентрации компонента в жидкости, данный метод проведения эксперимента может дать недостоверные результаты. Рандомизация, т.е. отбор проб жидкости с тарелок в случайной последовательности, даст более достоверный результат.

При экспериментальном исследовании влияния фрлегмового числа на работу ректификационной колонны необходимо также использовать рандомизацию. Последовательное увеличение флегмового числа в экспериментах может оказать влияние на распределение жидкости по тарелкам, в особенности повлиять на работу верхней тарелки, и внести систематическую погрешность в результаты экспериментов. Рандомизация будет плодотворной и в этом случае.

Рандомизация имеет большое значение не только при проведении естественнонаучных и технических исследований, но и в других отраслях науки: в медицине, социологии, образовании, политологии и др. Например, при проведении устного опроса в группе по изученной теме целесообразно подбирать вопросы и опрашивать обучающихся в случайной последовательности. Это даст более объективный результат.

\section{ЛИТЕРАТУРА}

1. Саутин С.Н. Планирование эксперимента в химии и химической технологии. Л., Химия, 1975, 48 с. 